\title{
The Migration of lons Along the Axis of a Polypyrrole Helix Channel in Aqueous Solution
}

\author{
David Antony Morton-Blake \\ School of Chemistry, Trinity College, Dublin, Ireland \\ Email address: \\ tblake@tcd.ie \\ To cite this article: \\ David Antony Morton-Blake. The Migration of Ions Along the Axis of a Polypyrrole Helix Channel in Aqueous Solution. International \\ Journal of Computational and Theoretical Chemistry. Vol. 7, No. 1, 2019, pp. 35-48. doi: 10.11648/j.ijctc.20190701.16
}

Received: January 18, 2019; Accepted: March 6, 2019; Published: April 1, 2019

\begin{abstract}
In order to investigate the passage of ions through a confined space, two substituted derivatives of a polypyrrole chain are considered in a helical conformation which forms a linear channel. The two species of sidechain on the 3 position of the pyrrole rings differ in their electron-withdrawing power. The system's response to a $\mathrm{pH}$ change by deprotonation of pyrrole rings is discussed in terms of a quantum tunnelling of a proton between two sites at the pyrrole's $\mathrm{N}$ atom. A characteristic 'inversion time' is calculated for the mechanism and compared for the two derivatives. In an investigation of the possibility that ions generated by the $\mathrm{pH}$ changes may enter the channel, molecular dynamics (MD) calculations are performed to calculate the fluctuating electrostatic potential at points along the axis for the polypyrrole chains in aqueous solution. It is found that although the atoms of the bare two polypyrrole channels derivatives generate very different time-averaged potential profiles along the axis, their polarising effect on the water molecules reorients the dipoles so as to oppose the charges from the polymer chain. It is shown that the charges on the polypyrrole helix imposes a 'solvent structure' on the water dipoles in the channel. The MD shows that when an electric field is applied along the channel axis the hydrated $\mathrm{H}^{+}$is accelerated to a point half way along the channel, where further progress is inhibited; however the passage of the $\mathrm{OH}^{-}$is unimpeded. These results are discussed in relation to the molecular confinement of the migrants through the axial electrostatic potentials and the migrant charges and structure.
\end{abstract}

Keywords: Molecular Dynamics, Molecular Channels, Helical Polypyrrole, Electrostatic Potentials, Hydrated Ions, Ion Migration, Proton Tunnelling

\section{Introduction}

A chemical system in which a species enters a molecular channel and maintains its motion, has an interest other than the analogous effects in, for example, the passage of ions through biological channels in membranes, which plays an essential role in the functioning of living cells. Vital as this aspect of molecular biology is to the subject, a chemist should be more excited by its importance in the design and synthesis of nano-systems which can achieve goals by accomplishing equivalent atomic-scale activity to create challenging novel devices.

While the transfer of ions between environments which are separated by a membrane plays an obviously vital role in the behaviour of living cells [1], the action need not be limited to biological activity. The present work describes a role that a simple synthetic molecular channel may play in the control of the $\mathrm{pH}$ of a system. It investigates the encounter of ions with a channel for the purpose of ascertaining the response by a controlled nano scale environment [2]. The architecture chosen for this purpose is based on a regular polypyrrole helix; the structural regularity of the main chain oligomer imposes an axial periodicity on the channel which facilitates the study of factors that may influence the dynamics of the molecular system.

Helical conformations may be found in polyheteroaromatic $\pi$-conjugated polymers. Polypyrrole has been predicted to respond to a twist around the inter-pyrrole bonds by forming a helical conformation in the neutral state [3]. Some workers have induced helical chains in the polymer by a chiral inducing method, producing polypyrroles with a good electromagnetic protecting abilities [4]. Helical structures of thiophene and polypyrrole polymers have been synthesized and characterised $[4,5]$. They form tight helices with small 
torsional angle $\varphi$ from the all-planar form as depicted in Figure 1(a). Helical polypyrrole has been proposed for medical applications [6].

These electroactive chains are rendered water soluble by polar side groups in the 3 position of the pyrrole ring. This work will consider polymer chains which have been chemically modified by the anion side chain thienyloxyethanesulphonate, $\quad-\mathrm{CH}_{2}-\mathrm{O}-\mathrm{CH}_{2}-\mathrm{CH}_{2}-\mathrm{SO}_{3}{ }^{-}$ and cation side chain thienyloxyethanetrimethylammonium $\mathrm{CH}_{2}-\mathrm{O}-\mathrm{CH}_{2}-\mathrm{CH}_{2}-\mathrm{NMe}_{3}^{+}$[7]. The species with these sidechains will hereafter be denoted $[\mathrm{S}]$ and $[\mathrm{N}]$ respectively. The work will examine polypyrrole chains which have been functionalised by this pair of side chains. In addition to the substituents' net charges whose effects on the material can be explored, we can also exploit the ability of the polypyrrole chain (in virtue of being an electroactive polymer) that it can undergo redox and other changes, thereby acquiring electronic conductivity and exhibiting interesting properties [8].

\section{Polypyrrole Channels}

The common conformation of oligomers and polymers of pyrrole and thiophene rings is that in which the heteroatoms in the monomer ( $\mathrm{N}$ or $\mathrm{S}$ ) are connected in an anti fashion so that they are alternately 'up' and 'down' along the main chain as shown in Figure 1(a). If a uniform torsion $\varphi$ is applied around each inter-pyrrole CC bonds then the resulting polymer chain assumes the helical conformation shown in parts (b) and (c) of the Figure. This conformation reflects the tendency of a wide range of regular polymers to twist around the bonds between the monomer units [9] the resulting helices of polythiophene and several of its derivatives have been observed in the solid state in electron microscopic investigations and uv spectra [10]. We are interested in the ability of this molecular channel to accommodate hydrated protons and hydroxide ions that are formed by $\mathrm{pH}$ control of the overall system.

This work reports molecular dynamics (MD) calculations on helical channels of both [S] and [N] modifications of polypyrrole. The helix that is simulated comprises 60 pyrrole rings forming a channel of length $60.5 \AA$ with six strands. The pitch of the helix (the separation of adjacent strands) is $7.2 \AA$ and its internal diameter is 8.8 or $10.5 \AA$ (depending on the pair of atoms selected for its definition.). Out of the 2732 water molecules in the aqueous system $65-70$ of them are within the water channel but which, during an MD run may drift between the channel and the exterior aqueous bulk. $\mathrm{Na}^{+}$ or $\mathrm{Cl}^{-}$counterions remain in a loose association with the polyelectrolyte chain, reducing the electrostatic repulsion between the monomer units [11]. The thermal motions of the solvent molecules may cast them adrift from these sites but throughout the MD they remained in the vicinity of the chain ends.

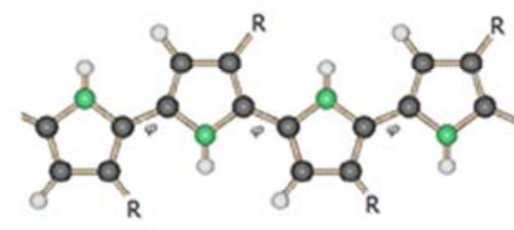

(a)

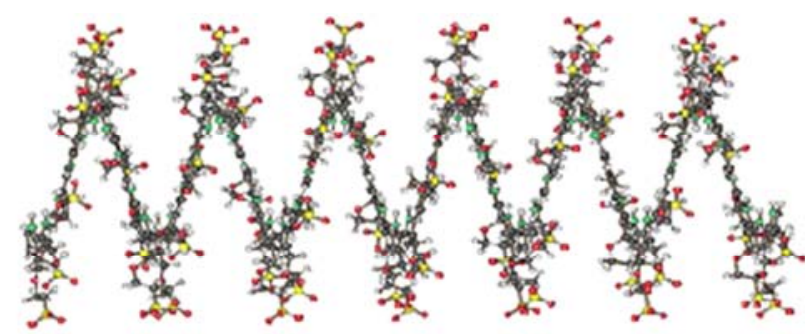

(b)

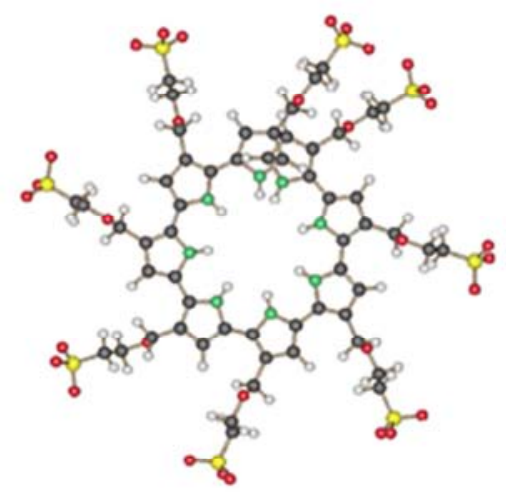

(c)

Figure 1. Two conformations of polypyrrole differing in the torsional angle $\phi$ about the inter-ring CC bond. (a) anti form where $\varphi=180^{\circ}$ and $(b)$ helix, $\varphi=5^{\circ}$. The substituent $R$ in the ' 3 ' position of the pyrrole ring is a sulphonate chain defined later in the text. part (c) is a view of (b) along the helical axis. 


\section{Tautomerism and Deprotonation in Pyrrole Units}

Notable features shared by this type of hetero-ring polymer and homogeneous hydrocarbon $\pi$ chains are their redox properties which are responsible for their electroactivity. For example the related compound porphyrin in Figure 2 and many of its tautomeric forms and compound have only approximate 4-fold symmetry [12] as can be seen from the

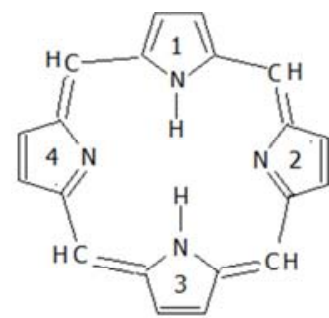

(a)

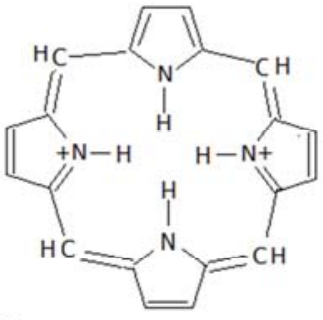

(b)

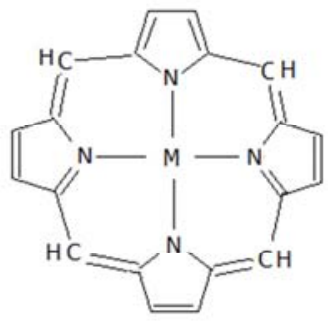

(c)

Figure 2. Three forms of a porphyrin (a) free base, (b) diacid and (c) a metalloporphyrin.

The resulting 'lability' of the $\mathrm{N}-\mathrm{H}$ bonding in the schemes depicted in Figure 2 suggests how defects might be expected to occur in polypyrrole involving the nitrogen and its electron lone pair. This follows from tautomeric changes in the bonding involving the $\mathrm{N}$ and $\mathrm{H}$ atoms which involves a tunnelling description of the two central $\mathrm{H}$ atoms in Figure 2(a). In a theoretical approach Maity et al. [13] investigated rate constants for the changes in the free base structure when the two central $\mathrm{H}$ atoms migrate to corresponding positions on the other pyrrole rings, and found that the changes are favoured by stepwise (rather than concerted) mechanisms. Hmigration causing tautomerism in porphyrins has been studied spectroscopically [14] and a ${ }^{15} \mathrm{~N}$ NMR investigation [15] studied the tunnelling migration of the $\mathrm{NH}$ proton in polycrystalline porphyrin-like compounds and established energy barriers. NMR work concludes that while the dynamics of the $\mathrm{H}$ migrations are fast on the NMR timescale, is easily observed at low temperatures. That the mobility is significantly reduced on deuteration of the $\mathrm{NH}$ group suggests that there is an important tunnelling component in

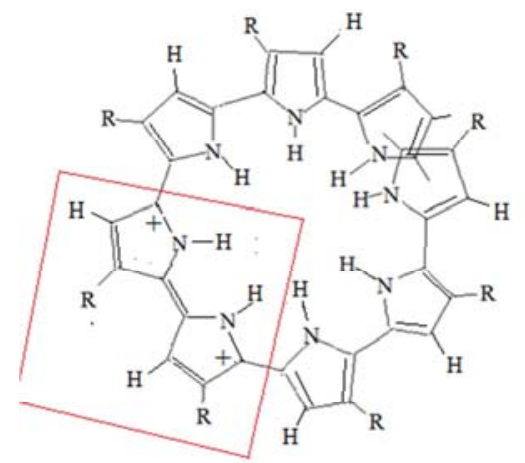

(a)
Lewis bonding structures of the free base in part (a) and the protonated form in part (b). Similarly its metallo compounds or chlorophylls in part (c) of the Figure (if the complexed atom $\mathrm{M}$ is magnesium) are also not consistent with equivalence of its four pyrrole units. The Figure shows that the breaking of the 4-fold symmetry in (a) and (b) can be traced to the atomic or oxidation sites of the amino $\mathrm{H}$ atoms which require for this purpose to be mobile.

the migration. The lability of the $\mathrm{H}$ atoms help to explain the characteristic biochemical and optoelectronic properties of these compounds.

The helical polypyrrole chain depicted in Figure 1(c) has its amino bonds $\mathrm{N}-\mathrm{H}$ directed towards the helical axis in a molecular arrangement that is rather like that in the porphyrin molecules in Figure 2 (a) and (b); the amino $\mathrm{H}$ atoms may thus be suspected of having a labile character as in the porphyrins. The effects of $\mathrm{pH}$ changes of aqueous polypyrrole solutions on the electrical conductivity of the solutions have been investigated [16]. These studies showed that there were $\mathrm{pH}$ ranges over which the $\mathrm{N}-\mathrm{H}$ group would undergo protonation or deprotonation. The consequent changes to conductivity led to the conclusion that $\mathrm{H}^{+}$or $\mathrm{OH}^{-}$ ions were occupying intercalation sites between the pyrrole rings. The concept of 'proton-motive force' by Mitchell and its use to describe the powering of biological components to generate the energy-rich fuel ATP [17] creates an interest in the study of systems that produce streams of protons under controlled conditions.

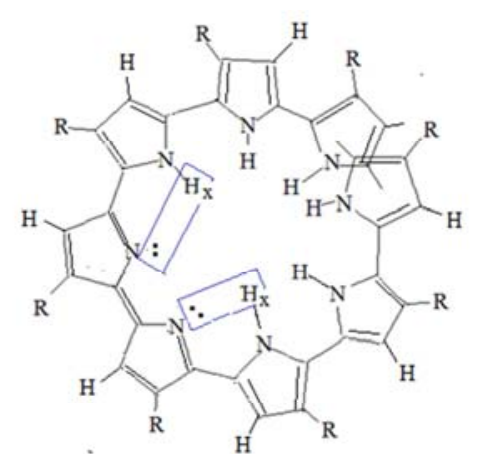

(b)

Figure 3. A single turn of the polypyrrole helix as in Figure 1(c) in which in part (a) a dipolaron (charge +2$)$ extends over two rings (boxed). Part (b) shows the result of double deprotonation from the red box in (a) which creates a neutral polymer chain with electron lone pairs on the two $N$ atoms. 
Figure 3(a) shows the result of a partial oxidation mechanism which has created a spinless two-ring dipolaron whose two +1 charges are separated by the width of a quinoidal pyrrole ring shown in the red boxed area. Such dipolarons, and ones consisting of more than two pyrrole rings, have been detected spectroscopically and discussed in the literature [18]. Their creation of mid-gap energy states has been associated with polypyrrole's electrical conductivity.

The dipolaron species shown here is the one with the shortest charge separation (lowest coulombic energy) and is stabilised by the polar electrolyte medium. In the film materials on which the $\mathrm{pH}$ investigations were conducted [16] the molecular structure in Figure 3(a) would probably be replaced by one in which the pyrrole rings are in anti conformations as in Figure 1(a). But in the helical chains that we are considering here Figure 3(a) shows that after deprotonation which converts the dipolaron in Figure 3(a) to the spinless neutral chain in Figure 3(b) there is a special transition region between the dipolaron and the kekuloid rings on either side of it. Here each $\mathrm{N}-\mathrm{H}$ bond in the region is adjacent to the lone pair of electrons borne by the neighbouring $\mathrm{N}$ atom in Figure 4 which depicts the polymer repeat segment. The interaction between the two adjacent pyrrole units reduces the distinguishability of the $\mathrm{N}$ atoms in the adjacent rings, presenting possible sites for the amino hydrogen atom $\mathrm{H}_{\mathrm{x}}$ in Figure 4 which shows nitrogen's deprotonated site as a red electron lone pair. The $H_{x}$ atom may move from a site on nitrogen a to which it is bonded to a second one on nitrogen $b$ which currently holds the lone pair (Figure 3(b)) and which is therefore available to form an alternative $\mathrm{N}-\mathrm{H}_{\mathrm{x}}$ bond. Since the labile $\mathrm{H}_{\mathrm{x}}$ atom should be associated with both $\mathrm{N}$ sites it may be considered to tunnel between the two sites.

Solution studies [16] on the polypyrrole derivatives have shown that an expulsion of protons from this structure occurring at $\mathrm{pH}>7$ results in the spinless neutral species depicted in Fig 3(b). Unlike the dipolaron the latter structure does not play a prominent role in conductivity, which is found to be significantly lower in basic solution. A conclusion drawn from these studies [16] was that following deprotonation the enhanced electrical conductivity observed in the acid $\mathrm{pH}$ ranges may be ascribed to the intercalation of $\mathrm{H}^{+}$or its hydrated form from the Figure 3(a) structure or other cations drawn from the aqueous solution.

The transition of the hydrogen atom between the two $\mathrm{N}$ atom sites in Figure 4 was monitored by performing quantum chemical computations on structures like that in Figure 4 with $\mathrm{H}_{\mathrm{x}}$ at different points between $\mathrm{N}(\mathrm{a})$ and $\mathrm{N}(\mathrm{b})$. Using a 6$111 \mathrm{G}(\mathrm{d})$ basis set Hartree-Fock calculations in the Gaussian package [24] were performed on the anionic [S] derivative depicted in Figure 4 and also on the corresponding [N] compound.

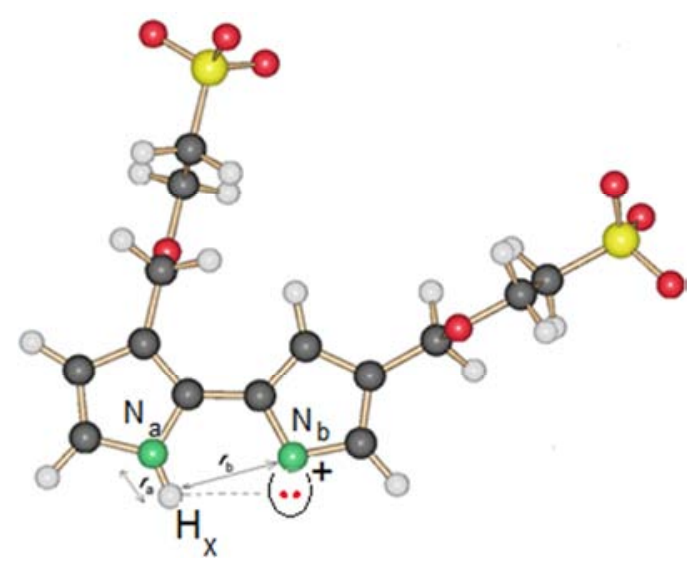

Figure 4. A bipyrrole portion of a polypyrrole chain containing the dipolaron shown in Figure 3. Each pyrrole unit has a sidechain that terminates in an $\mathrm{SO}_{3}{ }^{-}$group. The structure is shows an oxidised pyrrole ring with lone-pair electrons on the $N$ atom $b$ (red dots) and an adjacent pyrrole ring containing a nitrogen a and a migrating proton $H_{x}$.

The small mass of the hydrogen atom confers on it the property of unusual mobility as evidenced by its high mobility in lattices [19] and by the phenomenon of quantum tunnelling when the distance between protons or $\mathrm{H}$ atoms in a molecule is smaller than about the van der Waals separation. The latter behaviour has been studied in the hydrogenbonded dimers of carboxylic acids where the two acidic $\mathrm{H}$ atoms can be described on the simplest level by a singleparticle wave function extended over two low-energy zones [20]. Similar description are familiar for the hydrogen bonded associations of the moieties in helical ribonucleic acid chains [21, 22]. In the solid state [23] a particularly important instance of proton tunnelling is that occurring in the close hydrogen bonded configurations in various ice structures and in liquid water [5], contributing to its distinctive properties.

With a $6-311 \mathrm{G}(\mathrm{d})$ basis set a quantum chemical calculation was conducted on the [S] molecule in Figure 4. and its analogous [N] structure. In transition-state determining mode QST2 [24] the calculation monitored the migration of a proton $\mathrm{H}_{\mathrm{x}}$ from the site in which it formed a N-H bond with nitrogen atom a to that in which it formed one with nitrogen atom $b$ by employing the lone-pair electrons on the latter atom. The 'transition state' is that in which the proton is symmetrically half way along the trajectory and its position should constitute an energy barrier on the potential surface. Recalling that the side chains on the pyrrole rings are sulphonate $\mathrm{SO}_{3}{ }^{-}$or $\mathrm{NMe}_{3}{ }^{+}$derivatives as defined in Section 1 , and that the $\mathrm{H}_{\mathrm{x}}$ atom in the bipyrrole structure in Figure 4 is formally a proton, the molecular system in Figure 4 has an overall charge of $-1 e$ for the $\mathrm{SO}_{3}{ }^{-}$derivative in Figure 4. and $+3 e$ for the $\mathrm{NMe}_{3}{ }^{+}$structure.

The calculated potential energy profile in Figure 5 monitors changes as the proton undergoes unconstrained relaxation from the saddle-point on the potential energy surface (where its distances to the $\mathrm{N}$ atoms $\mathrm{a}$ and $\mathrm{b}$ are equal, and which has been determined to be a transition state in the 
complete trajectory of proton $\mathrm{H}_{\mathrm{x}}$ between $\mathrm{a}$ and $\mathrm{b}$ ) to its final state in which it forms the $\mathrm{N}_{\mathrm{b}}-\mathrm{H}_{\mathrm{x}}$ bond. For the $\mathrm{SO}_{3}{ }^{-}$ structure the energy barrier is $302 \mathrm{~kJ} \mathrm{~mol}^{-1}$ while that of the $\mathrm{NMe}_{3}{ }^{+}$compound the energy is $160 \mathrm{~kJ} \mathrm{~mol}^{-1}$. The substantially lower barrier for the ammonium derivative may be explained by its overall +3 charge, which would be expected to favour the departure of the proton from the $\mathrm{N}-\mathrm{H}$ group. Both barriers are substantially larger than the those that commonly give rise to 'inversion doubling' effects.

$\mathrm{H} \mathrm{N}$ distance

$$
r_{\mathrm{a}} \text { or } r_{\mathrm{b}}(\AA)
$$
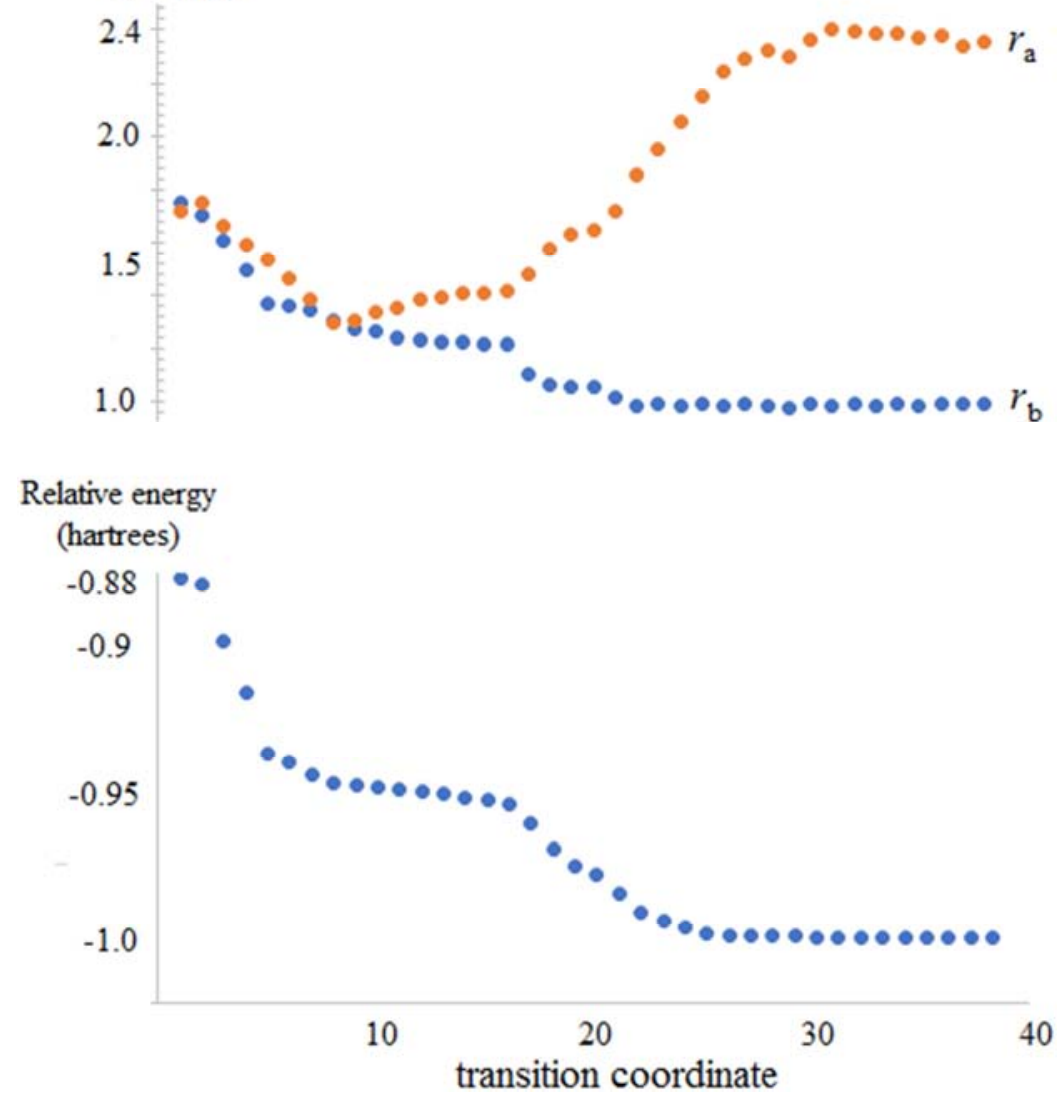

Figure 5. Transition between the atomic configurations from one in which $H_{x}$ is at the midpoint of $N$ atoms a and $b$ in Figure 4 to that in which it forms a 'natural' bond with b. Upper: the calculated progression of the changes in the $r_{a}$ and $r_{b}$ distances (Figure 4). Lower: the energy profile for the transition. The transition coordinate axis is common to both figures.

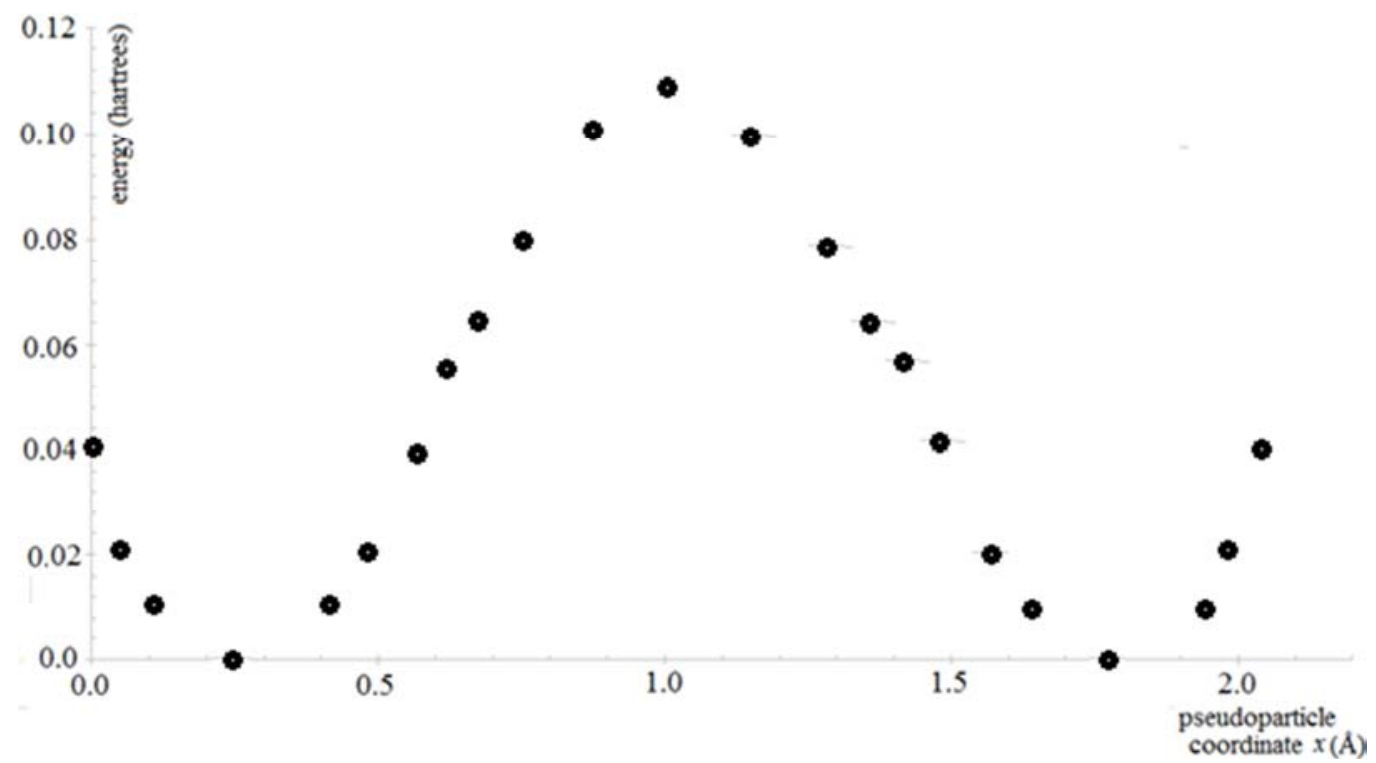

Figure 6. Potential profile fitted to the data in Figure 5. 
Several authors, e.g. $[25,26]$ provide algorithms to calculate the wave functions of a pseudoparticle in a quartic, one-dimensional double well potential. A treatment by Jelić and Marsiglio [25] uses the infinite-potential square well functions

$$
\psi_{n}(x)=\sqrt{\frac{2}{w}} \sin \left(\frac{n \pi x}{w}\right)
$$

(where $n=1,2,3, \ldots$ ) for each component well with width $w$, and is defined by eq. (1) in the displacement coordinate is in the range $0<x<L$ where $L$ is the effective width of the overall double well. The position of the maximum of the $V(x)$ potential (i.e. that of the barrier) is halfway along the double well at $x=1 / 2 L$. The energies $E_{n}$ that are associated with the tunnelling proton of mass $m_{p}$ in this square well model are

$$
E_{n}^{(0)}=n^{2} h^{2} / 8 m_{p} w^{2}
$$

where $w$ is the width of each of the two component wells which we fit to the energy profile of our proton migration calculation described above as $w=0.5 \AA$. The distance $g=$ $0.75 \AA$ is that from either of the two energy minima to the centre of the barrier at $x=1 / 2 L$ where we take $L=2.2 \AA$. Using these quantities, eqn. (2) gives the lowest energy level in the well of length $L$ to be $E_{1}^{(0)}=1.312 \times 10^{-20} \mathrm{~J}$.

The interactions between the quantum states in the component wells produce combinations $\psi=\sum_{n}^{\infty} c_{n} \varphi_{n}$ of the functions in (1) to construct Hamiltonian matrix elements

$$
\mathrm{H}_{\mathrm{nm}}=E_{n}^{(0)} \delta_{\mathrm{nm}}+\frac{2}{L} \int_{0}^{L} \varphi_{n}(x)^{*} \mathrm{~V}(\mathrm{x}) \varphi_{m}(x) \mathrm{dx}
$$

of $\mathrm{H}$ which is diagonalized to give the first order energies as its eigenvalues.

For the $[\mathrm{S}]$ and $[\mathrm{N}]$ structures the $V_{0}$ values are respectively $4.58 \times 10^{-19} \mathrm{~J}$ and $2.426 \times 10^{-19} \mathrm{~J}$. If each component well is taken to simulate that in a harmonic oscillator the lowest resonance then from (2) $\hbar \omega=$ $3.94 \times 10^{-20} \mathrm{~J}$. Parameters were taken from the double-well potential profiles described above, and the algorithms for the matrix elements $H_{n m}$ for in eqn. (3) that are provided by Jelić and Marsiglio [25] for the quartic potential $V(x)$ in their equation (A.17). Diagonalization of the resulting matrices $\mathrm{H}$ showed quantum levels that were, for the lower energies, in closely-split pairs. Their values showed acceptable convergence as the order of $\mathrm{H}$ approached $100 \times 100$. The $[\mathrm{S}]$ compound, with the higher $V_{0}$, showed a splitting of $1.90 \times$ $10^{-22} \mathrm{~J}$ in its lowest level, or in frequency units $1.80 \times 10^{12} \mathrm{~Hz}$. For the $[\mathrm{N}]$ derivative, whose barrier was about half that of $[\mathrm{S}]$, the corresponding energy splitting and frequency were an order of magnitude higher, at $1.99 \times 10^{-21} \mathrm{~J}$ and $1.89 \times 10^{13} \mathrm{~Hz}$. The behaviour may be interpreted as quantum tunnelling between the proton sites at nitrogen atoms $\mathrm{N}_{\mathrm{a}}$ and $\mathrm{N}_{b}$ with characteristic rates given by the associated frequencies [25].

The frequencies for both the pyrrole derivatives considered $\left(2 \times 10^{12}\right.$ and $\left.2 \times 10^{13} \mathrm{~s}\right)$ imply times which are commensurate with the picosecond timescale that is found for the measured reorientation correlation time of water [30]. Consider the result of the deprotonation into the aqueous solution from the
two-N region described. Since Figures 1(c) and 3 the $\mathrm{N}$ atoms show that the process occurs in the helix channel region which is an aqueous environment. We may therefore expect that the protons and the hydroxide ions become solvated by the reorienting water molecules as will be discussed below, where we shall consider that the migrating proton, especially, is in its hydrated form. (Interestingly the calculated frequencies of the tunnelling particle do not change their orders of magnitude if $\mathrm{H}^{+}$is replaced by the more massive $\mathrm{H}_{3} \mathrm{O}^{+}$. This is because of the constant $V_{0}$ and decreasing value of the $V_{0} / E_{1}^{(0)}$ ratio that is used in the Jelić and Marsiglio equations [25] in the current range.)

\section{Molecular Dynamics}

The calculations were performed by the DL_POLY MD code of Smith and Forester [27]. The structure ([S] or [N]) is specified by the contents of an MD cell defining a pseudolattice comprised of the 60-pyrrole helical channel in an environment of about 3000 water molecules (including the channel interior) as was described in Section 2.

The electrostatic energy component of atom-pair interactions were evaluated by Coulomb's Law and implemented the Ewald method to include long-range charge interactions. The non-charge parts by standard force fields to calculate the bonded- and non-bonded pair potentials. These were provided by the parameters of the generic Universal Force Field (UFF), which uses the Lennard-Jones 12-6 equation [28]. The atom-pair potentials were terminated by a cutoff of $11.0 \AA$. Jorgensen's TIP3P model [29] was used to provide the force field for the water solvent which, since it had been formulated to simulate the hydration of the ions, did not require additional electronic polarization effects. Atomistic potentials for $\mathrm{Na}^{+}$were those of Åquist [33] and for $\mathrm{Cl}^{-}$were by Lee and Rasaiah [34] which, in both cases were derived from aqueous solution data. Potential functions for all heteroatomic pairs were calculated by the LorentzBertholet geometric-mean combination rule [31].

The molecular geometries (bond angles and lengths) of the chemical species were used where the information was available; in the other cases the Gaussian package [24] was deployed to conduct quantum chemical computations using a $6-31 \mathrm{G}(\mathrm{d}, \mathrm{p})$ basis set. The geometry-optimised structures were matched with the results of $\mathrm{x}$-ray diffraction investigations on molecules and components that were as similar as possible to those of interest here were found. These were in turn compared with those of the Hartree-Fock or Density Functional calculations to construct the appropriate molecular geometries.

Quantum chemical calculations were also performed to obtain the partial atomic charges for which are required for the Coulomb contributions. The definition of the charges were the natural population analysis (NPA), whose use in predicting $\mathrm{pK}_{\mathrm{a}}$ values has been shown to be satisfactory [32]. In the case where a chemical species was modelled by a smaller molecule for a quantum calculation, adjustments 
were made for the 'dehydrogenation process' to restore the original structures. The molecular dynamics was conducted at standard pressure and temperature. With a timestep of 1 femtosecond $\left(10^{-15} \mathrm{~s}\right)$ the MD runs to achieve a thermally relaxed system were for $10^{6}$ (or in one case $10^{7}$ ) timesteps, but for some purposes such as tracking migrations considerably shorter runs were used. A Nosé-Hoover algorithm coupled the simulated system to room temperature and atmospheric pressure with thermostat and barostat relaxation times of 1 and 4 ps respectively.

\section{Channel Axial Potential}

Before turning to the behaviour of the ions in the channel it is instructive to see how the MD can describe the changes that permit the motion. Since forces governing the channel migration of the ions are largely derived from the electrical nature of the channel-migrant system [35] it was decided to calculate the electrostatic potential along the axes of the two chemical channels. These were derived from the atomic charges of the atomic constituents and their separation from a series of points along the channel axis after the MD system (channel and solution) had relaxed for a million timesteps. If atom $i$ lies respectively at axial and radial separations $z_{i}$ and $r_{i}$ from a point along the channel's $z$ coordinate and has a charge $q_{i}$ the potential at the point monitored is

$$
V(z)=\sum_{i}^{\text {atoms }} \frac{q_{i}}{4 \pi \varepsilon_{0} \sqrt{r_{i}^{2}+z_{i}^{2}}}
$$

where the summation may occur over all the atoms (channel and solvent) of the system. $r_{i}$ Since the environmental medium is an aqueous one, with a high dielectric constant, it would be interesting to ascertain the role of the constituent water molecules when the axial potential $V(z)$ is monitored. Reference to Figure 1(c) shows that substituting the 3 position of the pyrrole ring with the $[\mathrm{S}]-$ or $[\mathrm{N}]$ - based side chains deprives the helical chain of a centre of symmetry. The effect of the consequent electric dipole moment which is imposed on the channel is revealed in the asymmetry of the $V(z)$ traces in Figures 7(a) and 7(b), which are respectively for the helices with the two species of sidechain.

A comparison of the figures show that the contribution to the potential from the atoms of the helical channel alone (orange plots) is mainly in the negative region of the $V(z)$ axis for the anionic [S] compound but has positive values, numerically larger, for the cationic $[\mathrm{N}]$ species. This shows that, considering just the channel atoms, the potential on the axis is sensitive to the nature of the charge at the tips of the side chains. Both reveal the effect of the six strands of the helices (see Section 2) whose translational symmetry has been compromised. The continuity of their traces shows that over the million timesteps of the MD run the fluctuating field that would expected be from the motion of the atoms has averaged to produce smoothly varying potentials.

The blue traces are the $V(z)$ obtained when the contributions from the atoms of the channel and of all the water molecules were included. Their scattered nature shows that in this case the million timesteps do not average out the motions of the water molecules, which have apparently become unevenly bound to various atomic sites in the channel, resulting in the 'spikes' in both parts of the Figure. That these occluded water molecules are principally those which are inside the channel is revealed by a comparison of the yellow and grey plots. The former, which monitors the axial potential when the contribution of the in-channel water molecules is suppressed, is smooth, showing motional averaging. The grey plotted points, which monitor the electrostatic potential supplied by the water molecules that are within the channel are scattered as expected for fewer molecules that are confined to a space in which they associate with the charged atoms.

Significantly, both (a) and (b) show that despite the difference in the charge nature of the two species, when all the surrounding atoms are taken into account, the electrostatic potential along the channel axis of both species is mainly positive. This can be understood from the greater positive electrostatic contribution of the dry channel atoms of the $[\mathrm{N}]$ than of the negative $[\mathrm{S}]$ species.

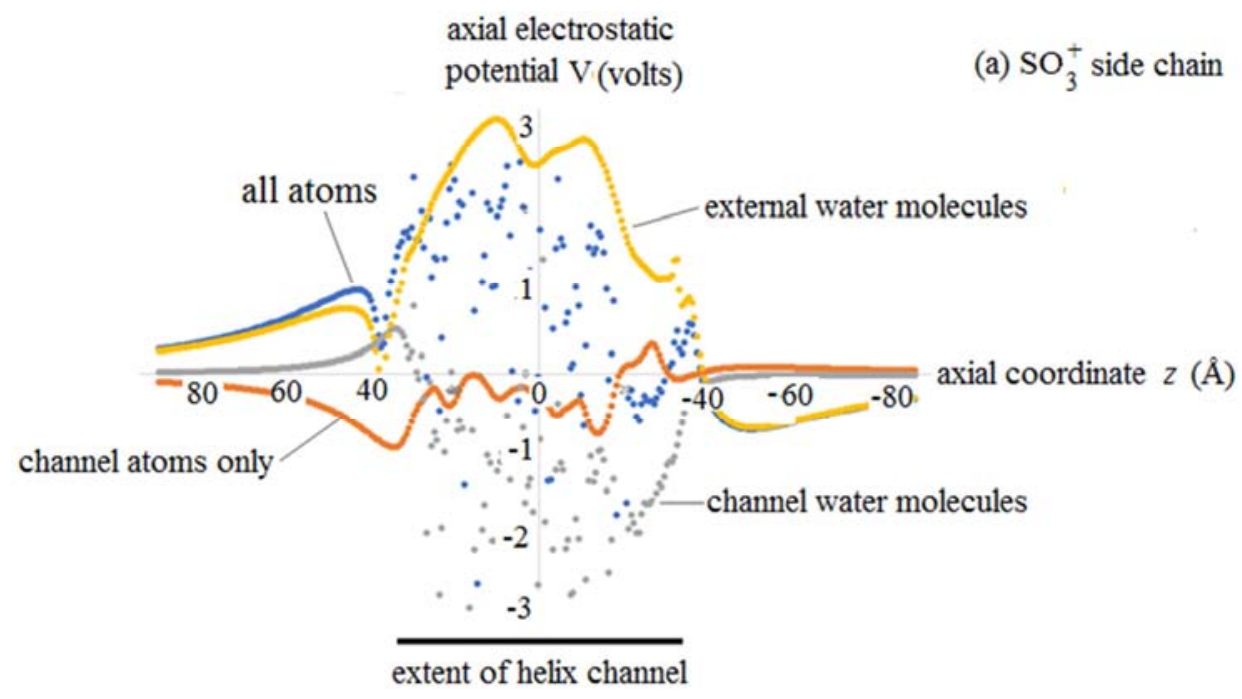




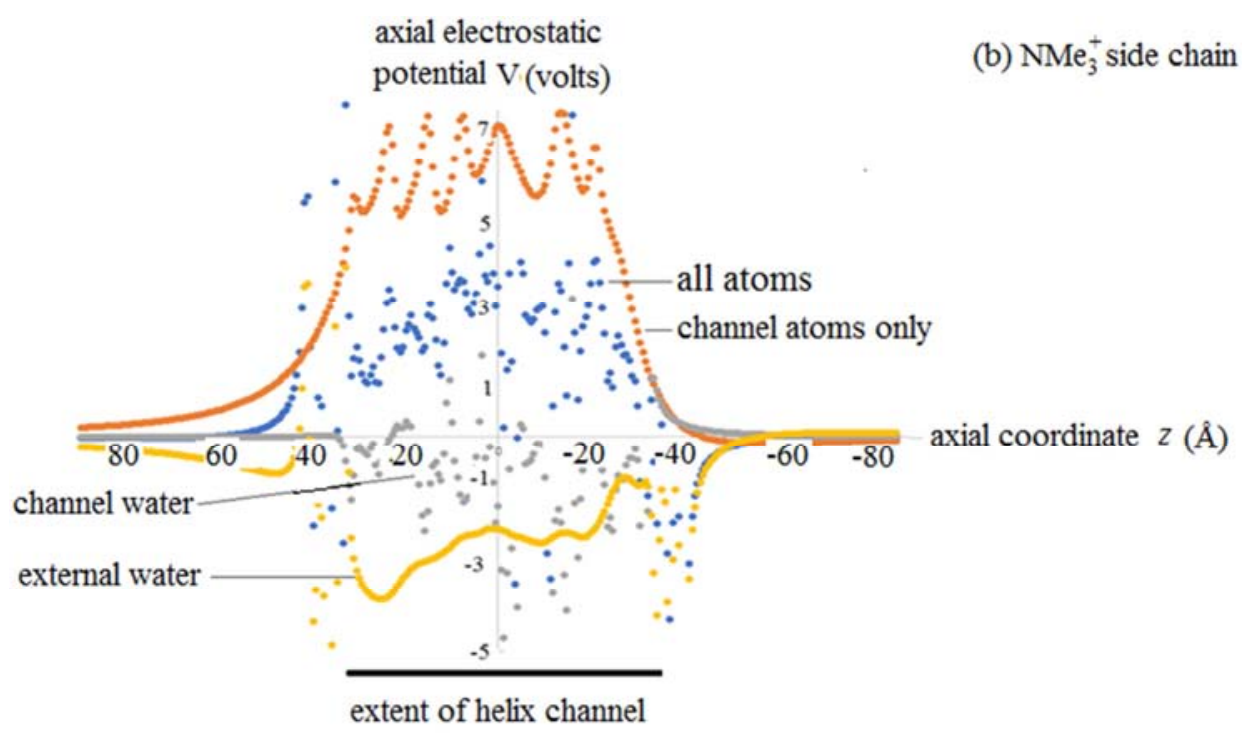

Figure 7. Electrostatic potential profiles along the axes of the polypyrrole channel for the (a) the [S] and (b) the [N] derivatives. The traces are labelled to indicate the atoms are invoked as components contributing to the potential.

Since the dimensions of the bare channel helices are almost equal in the two species the different behaviour of the water molecules in the two regions is due to their response to the different electrostatic charges of the channel atoms. Both (a) and (b) of Figure 7 show that the electric dipoles of the water molecules that surround the helix are polarized by the channel charges so that their electrostatic potentials are opposite to those of the dry channel. The in-channel water molecules show similar, though less pronounced, behaviour.

The axial potentials calculated show similar results for both the $[\mathrm{S}]$ and $[\mathrm{N}]$ chains, both of them monitoring overall positive potentials. Accordingly we select one of them [N] to constitute the ion channel for the remainder of this work.

\section{Channel Migration of the Ions}

Let us recall the chemistry of the polypyrrole chain, where as described in Sections 2 and 3, solution studies have shown ionic behaviour that depends on the $\mathrm{pH}$ of the aqueous medium, resulting in the generation of hydrated proton and hydroxyl ions. Although the remit of the present work is the behaviour of the former which are generated by the acidbased deprotonation of the polypyrrole chain $\mathrm{OH}^{-}$anions are also included since they would be present in the aqueous medium at higher $\mathrm{pH}$ ranges. With an interest in the possibility of their ion conduction through the polypyrrole channel, MD simulations were performed on the ions in an aqueous medium that contained the polypyrrole molecule responding to an electric field applied along the channel axis. While the fields $\varepsilon$ which were of order 0.1 volts $\AA^{-1}$ do not represent a specific physical quantity in the simulated molecular system, this procedure of boosting the ion motion is deployed since the times involved in the migration are considerably longer than those that can currently be used in current MD simulation. (Nevertheless simple calculations readily show that short range electric fields of this order and greater do indeed occur in the neighbourhood of ions in solution.)

The molecular geometries and atomic charges of the migrant ions required are obtained from Hartree-Fock 6$311 \mathrm{G}(\mathrm{d})$ calculations [24]. The hydrated proton that is created in the deprotonation step is subject to uncertainties in its nature and structure. Several studies have been conducted to explain its anomalously high conductivity in aqueous solution. The cations proposed by Zundel [36] and by Eigen et al. [37] consist of a basic $\mathrm{H}_{3} \mathrm{O}^{+}$(hydronium) unit which is solvated to different extents by water molecules. An infrared study [38] has characterised smaller units $\mathrm{H}_{7} \mathrm{O}_{3}{ }^{+}$in which three waters solvate a hydronium cation. Proton conductivity is proposed to occur by a dynamic re-conformation process permitting the transfer or tunnelling of a $\mathrm{H}^{+}$ion to an adjacent water molecule. The environment of the liberated proton considered in the present work differs from that in the cited investigations since the cation is subject to spacial confinement in the channel of a helical chain whose axial radius is $4.5 \AA$. If there is to be channel migration the cation in this situation would be smaller than those in the cited works [36, 37]; consequently the $\mathrm{H}_{3} \mathrm{O}^{+} \cdot \mathrm{H}_{2} \mathrm{O}$ species was selected as the migrant cation. This structure was optimised by a Hartree-Fock 6-311G(d) calculation [24] to give a nonplanar configuration with a linear $\mathrm{O}-\mathrm{H}-\mathrm{O}$ axis and bond lengths and angles $(13)=1.00 \AA,(34)=1.50 \AA,(132)=$ $114.7^{\circ}(137)=110.3^{\circ}$. The NPA charges were calculated to be $q\left(\mathrm{H}^{1}\right)=+0.5375 e, q\left(\mathrm{O}^{3}\right)=-0.8587 e, q\left(\mathrm{H}^{4}\right)=+0.5676 e$.

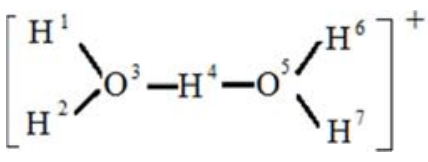

Figure 8. The $\mathrm{H}_{3} \mathrm{O}^{+} . \mathrm{H}_{2} \mathrm{O}$ structure.

The hydrated $\mathrm{OH}^{-}$ion is not as well characterised as the hydrated proton and although structures have been proposed, including one that is similar to the the Zundel one for $\mathrm{H}_{5} \mathrm{O}_{2}{ }^{+}$ [36], a IR study finds that they are unstable, that the 
hydrogen bond in hydrated $\mathrm{OH}^{-}$is considerably weaker than that in the hydrated proton [39] and that it would dissociate within a few vibration periods. Thus for the present work the model used is that of an $\mathrm{OH}^{-}$anion which forms hydrogen bonds with water molecules in accordance with Jorgensen's TIP3P model [29]. Hartree-Fock calculations described above provide a $\mathrm{OH}^{-}$ion a bond length of $0.9557 \AA$ and NPA charges $q(\mathrm{O})=-1.2775 e, q(\mathrm{H})=+0.2775 e$.

The charges of the channel atoms that were used to calculate the axial potentials for Figure 3(b) were obtained from a quantum chemical calculation on the $[\mathrm{N}]$ derivative of a deprotonated bipyrrole molecule in which the $\mathrm{N}$ of the pyrrole ring did not bear a $\mathrm{H}$ atom. Since the mechanism of the deprotonation event described in Section 3 and Figure 3 involves just two explicit protons this was the picture carried into the MD as the simplest mechanism for simulation of the deprotonation to be considered. Consequently it was conducted on a polypyrrole channel in which $\mathrm{H}$ atoms were removed from two of the pyrrole NH units, each left with a negative charge. (Corresponding deprotonations at lower $\mathrm{pH}$ of the bulk solution might naturally be described by roles which involve more than two protons.) The loss of protons required a compensation to form a charge-neutral system by the migrant ion described in the previous paragraph and by a positive charge provided by a non-migrating 'dummy' $\mathrm{H}_{3} \mathrm{O}^{+} . \mathrm{H}_{2} \mathrm{O}$ species in the aqueous medium. The facts that the atoms of these two pyrrole rings are more negatively charged than those of the remaining 'regular' pyrroles and that the missing $\mathrm{H}$ atoms clear more axial space for the cation to occupy may possibly influence the migrant's channel trajectory.

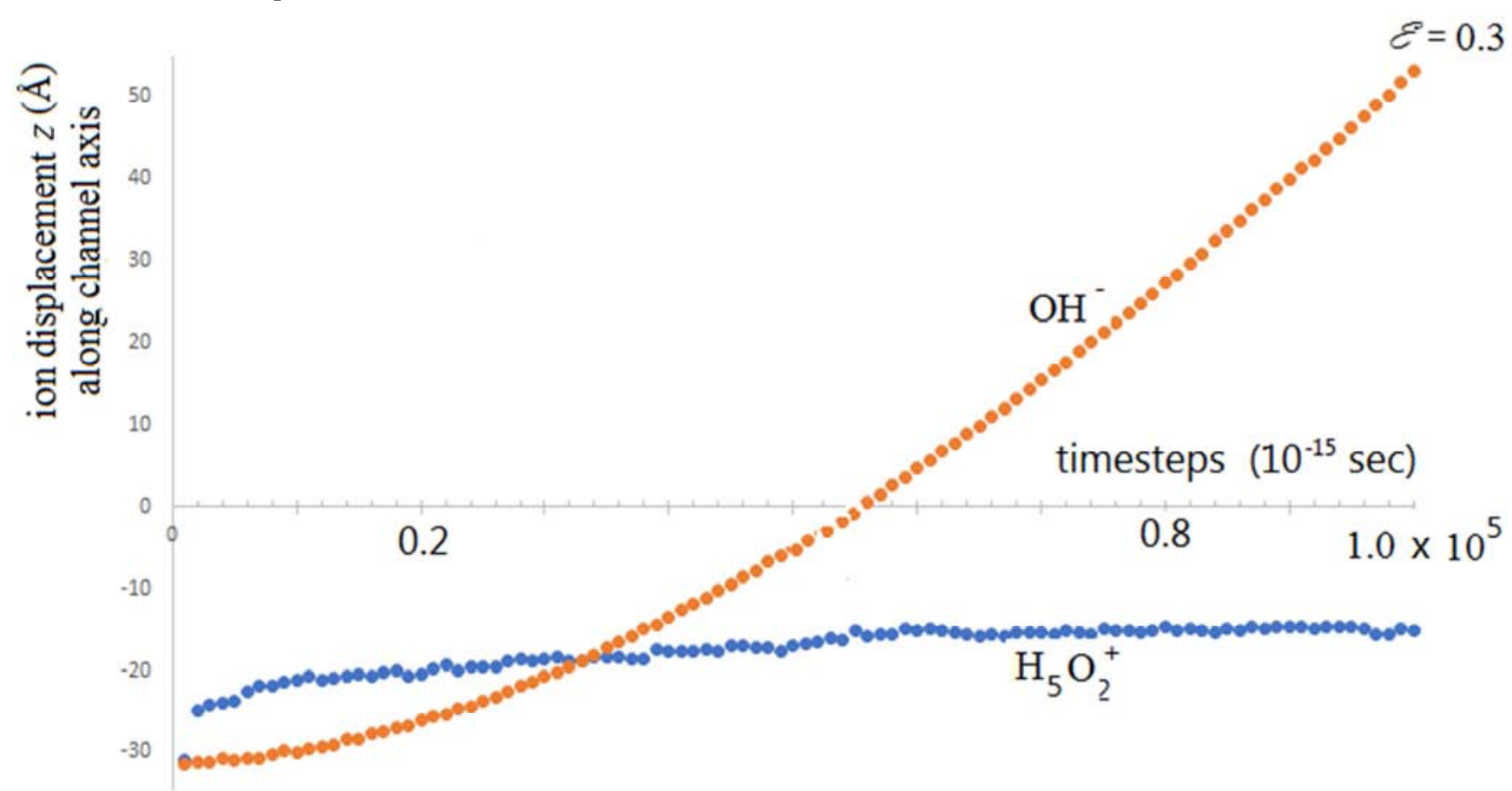

Figure 9. A comparison of the migrations of the channel migrations of the hydrated proton cation $\mathrm{H}_{5} \mathrm{O}_{2}{ }^{+}$and of the $\mathrm{OH}$ anion in an axial 0.3 electric field. (The field is applied in opposite directions for the two ions.).

With the $\mathrm{OH}^{-}$and $\mathrm{H}_{5} \mathrm{O}_{2}^{+}$migrants starting from the same axial site $z=31.0 \AA$ at the right-hand end of the channel in Figure 7), electric fields $\varepsilon$ of equal magnitude were applied separately in opposite directions ( 0.3 and -0.3 units $)$ to induce the two oppositely-charged ions to move in the channel. The result in Figure 9 shows that in their simulations for $10^{5}$ timesteps the $\mathrm{OH}^{-}$and $\mathrm{H}_{3} \mathrm{O}^{+} . \mathrm{H}_{2} \mathrm{O}$ migrant ions respond very differently to the fields. The speed of the $\mathrm{OH}^{-}$anion's trajectory along the along the $\sim 60 \AA$ long channel is almost unimpeded and is maintained after its exit from the end of the channel so that its motion is virtually the same as that in the bulk solution. The hydrated proton, on the other hand, after an initially rapid progress, shows that its migration is inhibited, and MD runs at several fields $\varepsilon$ as shown in Figures 10 and 11 only just bring the cation to positive $z$, i.e. the second half of the channel.

A possibility which should be tested is whether the ion's severe retardation is caused by its drift from the axial cavity of the channel into its walls, where steric forces from the polymer and side chain would impede its progress along $z$. However Figure 11, which plots both the axial and radial positions of the ion in parts (a) and (b) for a million timesteps, shows that since the radial coordinate $r(t)$ maintains a value $r \approx 4 \AA$, indicating that although it approaches the channel walls (radius $4.9 \AA$ ) it still remains within the axial cavity over the migration run. 


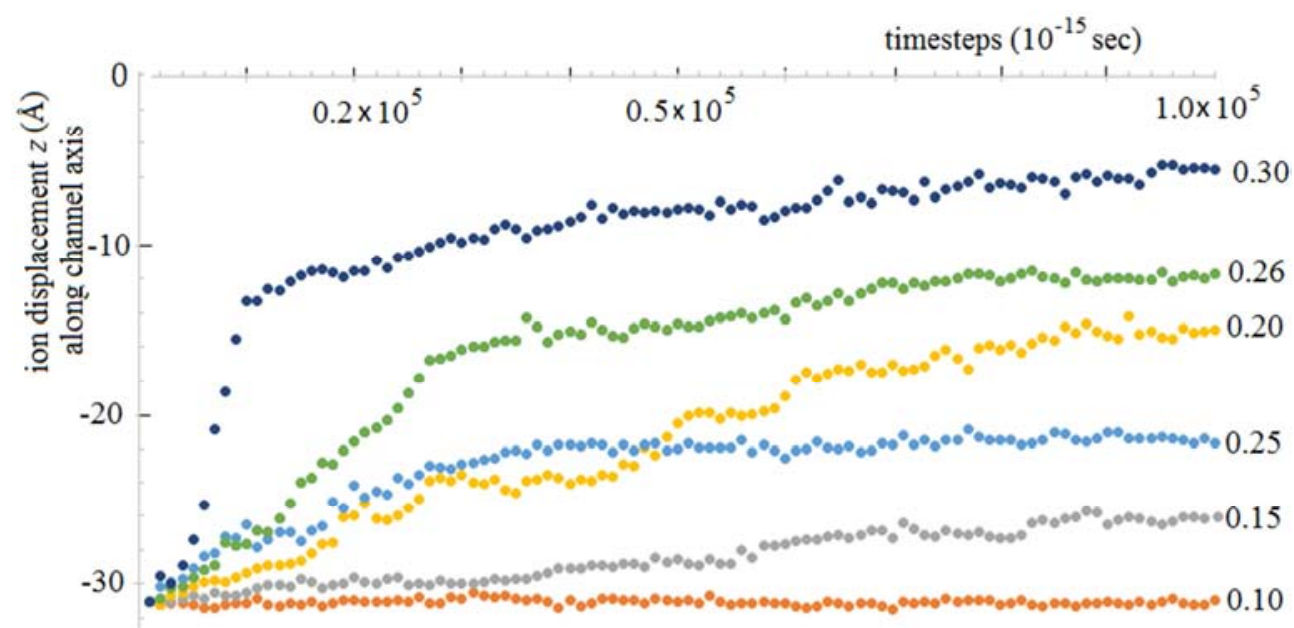

Figure 10. Migration of the $\mathrm{H}_{5} \mathrm{O}_{2}{ }^{+}$cation in various electric fields illustrating the 'fast' and 'slow' regions.

The rapid passage of the $\mathrm{OH}^{-}$ion from its initial position $z$ $=-3.1 \AA$ on the channel axis may be attributed to its small size and to the fact that after clearing the initial $V(z)$ spikes in Figure $7(\mathrm{~b})$ it accelerates to an axial region in which the electrostatic potential is mainly positive, producing a linear trace. As seen in the million-timestep trajectory in Figure 11, the same encounters have the opposite effect on the $\mathrm{H}_{5} \mathrm{O}_{2}{ }^{+}$ ion, which is initially speeded up by the negative $V(z)$ spikes and retarded by its passage into the positive $V$ in the second half of the channel.
Another factor influencing the slower cation's migration arises from the values of the radial coordinate referred to above, which shows departures from the channel axis of up to $4 \AA$, implying close approach to the nitrogen of the pyrrole rings. The calculated atomic charges given in Figure 11 for the pyrrole fragment of the $[\mathrm{N}]$ polymer unit imply that the departure of the $\mathrm{H}_{5} \mathrm{O}_{2}{ }^{+}$ion from the channel axis would bring it into a more negative electrostatic potential than that shown in Figure 7(b) which relates to the purely axial passage at $r=$ $0 \AA$. The issue is discussed in Section 9 below.

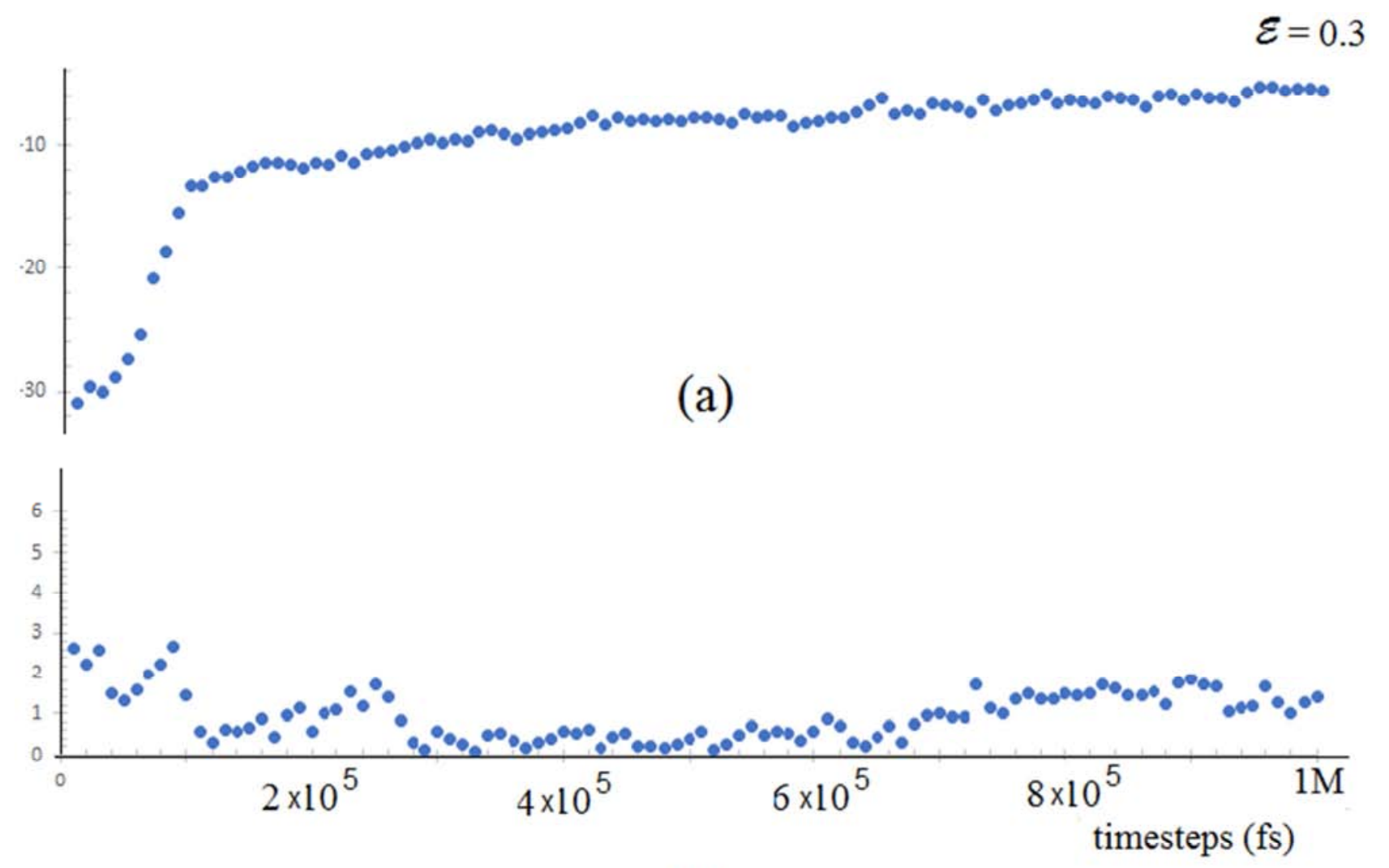

(b)

Figure 11. Axial electric fields applied respectively to a hydrated proton cation starting a migration from one end of a helical polypyrrole channel ( $z=30$ A). The trace in (a) monitors the axial position of the ion (z direction). Part (b) shows that the radial position of the $\mathrm{H}_{5} \mathrm{O}_{2}{ }^{+}$ion (being less than the channel's internal radius of 4.9 A) always stays within the channel. 


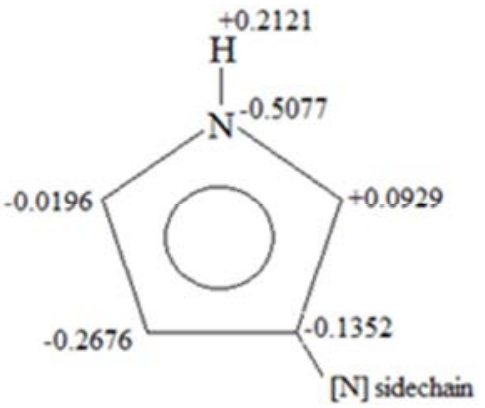

Figure 12. Calculated NPA charges on pyrrole ring. They reflect the loss of the twofold symmetry caused by the asymmetric chemical substitution of the polar [N] side groups.

\section{Thermally Activated Migration}

The axial migration of the $\mathrm{H}_{5} \mathrm{O}_{2}{ }^{+}$ion at temperatures $T$ between 290 and $340 \mathrm{~K}$ in Figure 13 show that the ion's displacement $z$ increases with temperature, suggesting that the migration is thermally activated. The $z(T)$ values were used to calculate $R$, the rate of increase of $z$ with temperature $T$ in the rapid and slow migration regions, taken at 6000 and at 29000 timesteps respectively. Slopes of the Arrhenius plots of $\ln R v s 1 / T$ from the inset to Figure 13 permit estimation of the activation energies as $5 \mathrm{~kJ} \mathrm{~mol}^{-1}$ and $25 \mathrm{~kJ} \mathrm{~mol}^{-1}$ for the fast and inhibited transport regions.

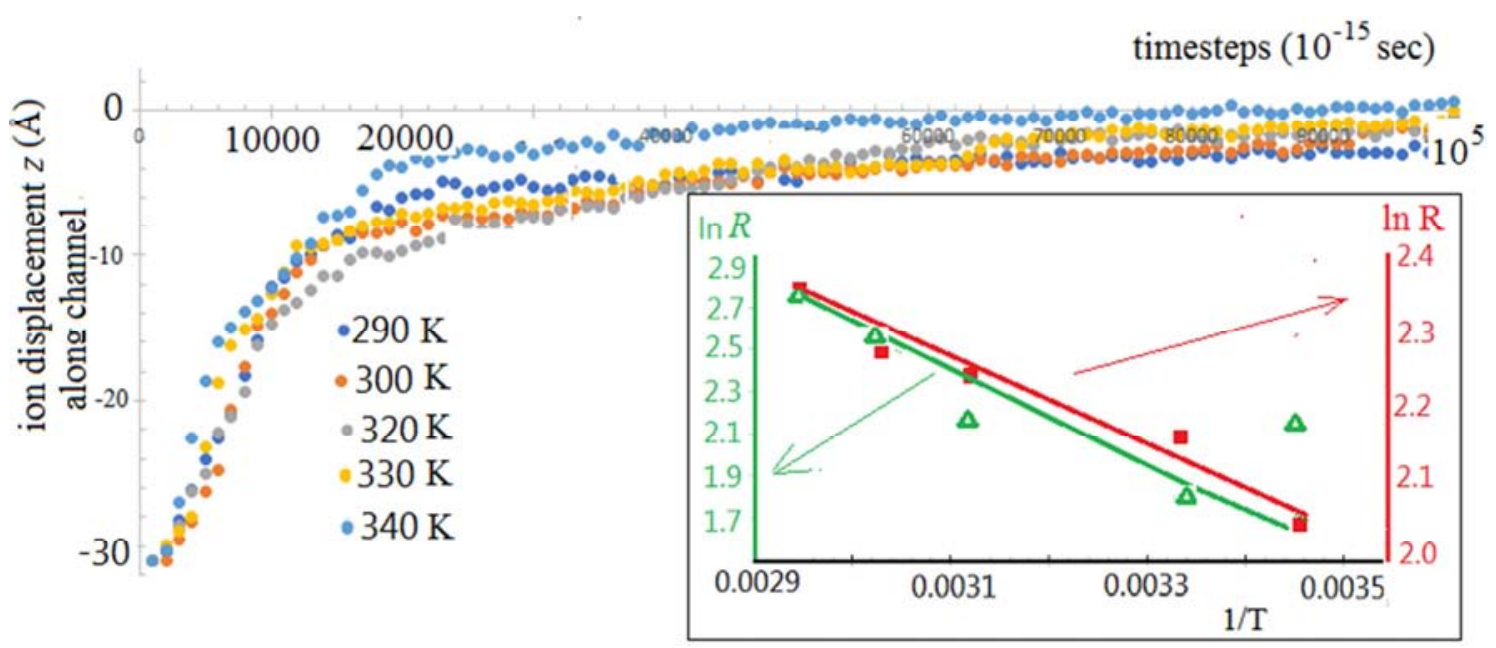

Figure 13. Main figure: $z(t)$ migration of the $\mathrm{H}_{5} \mathrm{O}_{2}{ }^{+}$ion in a $\varepsilon=0.3$ electric field at different temperatures for the fast and slow regions. $\square$ : Slow migration; $\triangle$ : Fast. Inset: Arrhenius curves of migration rates $R$ and temperature to evaluate activation energies.

\section{Channel Waters}

As solvent water molecules drift in and out of the channel in such a way that the channel preserves on average of 90 100 molecules during a $10^{5}$ or $10^{6}$-timestep run it would be interesting to ascertain information on whether they exhibit any form of liquid packing on entry into their new environment. An effect of spacial confinement on the water molecules inside the polypyrrole channel is shown in Figure 14, which (in the absence of an applied electric field) plots the frequency distribution of the mean angle $\theta$ between the twofold axis of the $\mathrm{H}_{2} \mathrm{O}$ molecule and the channel axis. The molecular axis is the direction of the water dipole and the Figure shows that over the $10^{5}$-timestep md run, the confining effect of the channel removes the random distribution of $\theta$ values expected for bulk water, producing ones in which they associate in intervals. The result suggests a tendency for the channel to induce a liquid lattice formation on the solvent molecules in the channel.

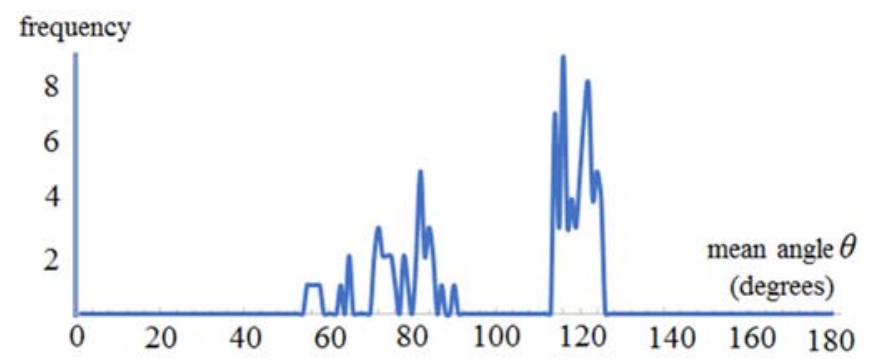

Figure 14. The distribution of the mean angle $\theta$ between the twofold axis of the $\mathrm{H}_{2} \mathrm{O}$ molecule and the channel axis.

\section{Discussion}

The picosecond characteristic time calculated in Section 3 for the tunnelling transfer of the proton and its hydrated form between adjacent $\mathrm{N}$ atoms in the pyrrole ring is slower than that of the $\mathrm{H}$ atom. The molecular dynamics of the subsequent sections thus permits us to use md to investigate the motion of the hydrate ion inside the channel. 
The work underlines the response of the solvent dipoles to the charges on the migrant channel. The subject of ions migrating in a confined space has spawned interesting investigations in industrial and fundamental contexts [40]. Some of these studies describe the motion after recasting the normal bulk-liquid equations describing the ion trajectories. Work in this laboratory has been concerned with the role played in ion selection by in-channel water molecules on the motions of the ions and the effects of their sizes and charges [41]; associated work considers the activation energies encountered by the ions when they shed their hydration shells to enter a molecular channel from the aqueous bulk [42]. Although in this presentation desolvation is not relevant since the hydrated hydronium ions were chemically generated within the channel (Section 3), the work has found regions of thermally activated motion for the hydrated proton after it embarks on its channel trajectory after departure from a starting point at the channel entrance.

The origins of the two trajectory regions of the $\mathrm{H}_{5} \mathrm{O}_{2}{ }^{+}$ion in the channel (Section 6), that are sharply divided by their activation energies), are not completely clear, and explanations that are based on time-averaged electrostatic distributions may be of limited validity. The dynamical behaviour is probably associated with the fluctuating electrostatic distributions within the channel, which arise from the atoms of the channel and of the water molecules, both inside and outside the channel. While these contributions are simulated in the molecular dynamics, they cannot reflected in the time-averaged potentials of Figure 7. If this conclusion is valid a future mathematical account of channel ion migration must involve the explicit time behaviour of the ion migrants, the channel and the water molecules.

\section{Conclusions}

The work finds that passage of the ions in the molecular channel depends on the charge and size of the migrant: a calculation of activation energies concludes that the smaller $\mathrm{OH}^{-}$anion experiences considerably less steric inhibition in its trajectory than the bulkier $\mathrm{H}_{5} \mathrm{O}_{2}{ }^{+}$cation. It is supposed that this is because the latter ions may more easily approach the 'walls' of the $9 \AA$ channel which impose a coulomb hindrance on their motion, which is reflected in a contribution to migrant activation energy. Indeed, some trajectories (not discussed) showed that ions were captured by the channel walls, preventing further channel migration. However, as discussed in the previous section future work should take into account the vibrational nature of the channel atoms, which is currently being addressed in this laboratory.

We have also determined a tendency for the solvent 'structure formation' imposed by the partial charges of the channel atoms on the water dipoles in the solvent which they surround. This would seem to be an important effect to consider in the functioning of a nanochemical tube or channel that involves the passage of aqueous ions.

\section{Computational Facilities}

These were provided by An Institúd um Theicneolaíocht Eolais agus Riomhfhorbairt na hÉireann (IITAC).

\section{Supplementary Data}

The author would be happy to forward data files for the atomic coordinates of the helical channels described in this work.

\section{References}

[1] S_H Chung, O. S. Anderson and V. V. Krishnamurthy (Eds.), Biological Membrane Ion Channels: Dynamics, Structure, and Applications (2007) Springer (2007); R. Pethig and D. B. Kell, 'The passive electrical properties of biological systems: their significance in physiology, biophysics and biotechnology', Phys. Med. Biol., 1987, 32 (8) 933-970 (1987).

[2] R. Ballardini, V. Balzani, A. Credi, M. T. Gandolfi, M. Venturi, 'Artificial molecular-level machines: which energy to make them work?’ Acc. Chem. Res. 34 445-455 (2001).

[3] X. Lin, J. Li, E. Smela and S. Yip 'Polaron-induced conformation change in single polypyrrole chain: An intrinsic actuation mechanism' Int. J. of Quantum Chemistry 102980 (2005).

[4] A. Xie, F. Wu, W. Jiang, K. Zhang, M. San and M. Wang 'Helical conducting polymers have good optical and electrical activities. Helical polypyrrole is synthesized by a chiral inducing method' J. Mater. Chem. C 52175 (2017).

[5] F. Garnier, G. Tourillon, J. Y. Barraud, H. Dexpert, 'First evidence of crystalline structure in conducting polythiophene' J. Mat. Sci. 202687 (1985); G. Tourillon and Y. Jugnet, 'Electronic and structural characteristics of five poly membered heterocycles (polythiophene, polypyrrole): An ultraviolet and $\mathrm{x}$ - ray photoelectron spectroscopy study', Journal of Chemical Physics 89, 1905 (1988).

[6] Y. Tadesse, R. W Grange and S. Priya, 'Synthesis and cyclic force characterization of helical polypyrrole actuators for artificial facial muscles', Smart Mater. Struct. 18085008 (2009).

[7] M. Chayer, K. Faìd, M. Leclerc, 'Highly Conducting WaterSoluble Polythiophene Derivatives', Chem. Mater. 9 29022905 (1997); É. J. Lemieux, M. Leclerc, 'Sensing via conformational changes of conjugated polythiophenes' (Chapter 7 pp 231-261) of Conjugated Polyelectrolytes: Fundamentals and Applications, Bin Liu, G. C. Bazan (Eds.) Wiley-VCH Verlag GmbH \& Co. (2013).

[8] D. A. Morton-Blake, 'Ion Selection by Redox Changes in a Soluble Helical Polythiophene Channel', Diffusion Foundations Vol. 9 pp 1-15 (Zurich 2016).

[9] (See for example discussions in) C. W. Bunn, E. R. Howell, 'Structures of molecules and crystals of fluorocarbons', Nature 174 549-551 (1954); A. Azri, P. Giamarchi, Y. Grohens, R. Olier, M. J. Privat, Polyethylene glycol aggregates in water formed through hydrophobic helical structures', Colloid interface Sci. 329 14-19 (2012). 
[10] G. Tourillon, F. Garnier, 'Morphology and crystallographic structure of polythiophene and its derivatives' Mol. Cryst. Liq. Cryst. 118 (1985) 221; R. Yang, D. F. Evans, L. Christensen and W. A. Hendrickson, 'Scanning tunnelling microscopy evidence of semicrystalline and helical conducting polymer structures' J. Phys. Chem. 946117 (1990); G. Caple, B. L. Wheeler, R. Swift, T. L. Porter, S. Jeffers, Scanning tunnelling microscopy of polythiophene, poly(3-methylthiophene) and poly(3-bromolthiophene), ibid 5639-5641; F. Garnier, G. Tourillon, J. Y. Barroud, H. Dexpert, 'First evidence of crystalline structure in conducting polythiophene' J. Mat. Sci. 20 (1985) 2687; R. D. McCullough, P. C. Ewbank, Regioregular head to tail coupled poly(3-alkylthiophene) and its derivatives, in T. A. Skotheim, R. L. Elsenbaumer J. R. Reynolds (Eds.), Handbook of Conducting Polymers, Dekker, New York (1998).

[11] O. Bernard and J.-P. Simonin, 'Association of counterions in polyelectrolytes: Thermodynamic properties in the binding mean sphere approximation' J. Mol. Liquids 970 14-24 (2018).

[12] E. B. Fleischer, 'The structure of porphyrins and metalloporphyrins', EB Fleischer - Accounts of Chemical Research, 3 105-112 (1970).

[13] D. K. Maity, R. L. Bell and T. N. Truong, 'Mechanism and Quantum Mechanical Tunnelling Effects on Inner Hydrogen Atom transfer in Free Base Porphyrin', J. Am. Chem. Soc. 122 897-906 (2000).

[14] E. A. Ermilov, B. Büge, S. Jasinski, N. Jux and B. Röder 'Spectroscopic study of NH-tautomerism in novel cycloketo-tetraphenylporphyrins', J. Chem. Phys. 130 1345092018.

[15] U. Langer, C. Hoelger, B. Wehrle, L. Latanowicz, E. Vogel and H.-H. Limbach, '15N NMR Study of Proton Localization and Proton Transfer Thermodynamics and Kinetics in Polycrystalline Porphycene' Journal of Physical Organic Chemistry 13 23-34 (2000).

[16] Q. Pei and R. Qian, 'Protonation and deprotonation of polypyrrole chain in aqueous solution' Synthetic Metals 45 35-48 (1991); J. Stejskal, M. Trchová, P. Bober, Z. Morávková, D. Kopecký, M. Vrńata, J. Prokeš, M. Varga and E. Watzlová 'Polypyrrole salts and bases: superior conductivity of nanotubes and their stability towards the loss of conductivity by deprotonation' RSC Advances 688382 (2006).

[17] P. D. Mitchell, 'The protonmotive Q cycle: A general formulation'. FEBS Letters. 59 (2): 137-139 (1975); Peter Mitchel's 1978 Nobel speech. The Nobel Prizes 1978, Editor Wilhelm Odelberg, [Nobel Foundation], Stockholm, 1979; N. Lane, Life: Inevitable or fluke? New Scientist 21 June 2012

[18] See for example ref [16] and) J.-L. Brédas, J. C. Scott, K. Yakushi and G. B. Street 'Polarons and bipolarons in polypyrrole. Evolution of band structure and optical spectrum upon doping' Phys. Rev. B 30 1023-5 (1984); P. Pfluger, G. Weiser, J. C. Scott and G. B. Street 'Electronic structure and transportin the organic "Amorphous semiconductor" polypyrrole' in 'Handbook of Conducting polymers' Vol. 2 (Ed. T. A. Skotheim) Dekker 1986; M. J. L. Santos, A. G. Brolo and E. M. Girotto 'Study of polaron and bipolaron states in polypyrrole by in situ Raman spectroelectrochemistry' Electrochim Acta 52 6141-5 (2007) and references theein.
[19] Eds. G. Alefeld and J. Völkl 'Hydrogen in Metals', Springer 1978.

[20] L. Evangelisti, P. Écija, E. J. Cocinero, F. Castaño, A. Lesarri, W. Caminati and R. Meyer, 'Proton tunneling in heterodimers of carboxylic acids: A rotational study of the benzoic acid -formic acid bimolecule', J. Phys. Chem. Lett. 3 3770-5 (2012).

[21] P.-O. Löwdin, 'Quantum genetics and the aperiodic solid. Some aspects of the biological problems of heredity, aging and tumours in view of the quantum theory of the DNA molecule'. Advances in Quantum Chemistry 2 Pp 213. Academic Press 1965.

[22] See for example A. D. Godbeer, J. S. Al-Khalili and P. D. Stevenson, proton tunnelling in the adenine-thymine base pair'. Phys. Chem. Chem. Phys. 17 13034-44 (2015).

[23] D. F. Brougham, R. Caciuffo and A. J. Horsewill, 'Coordinated proton tunnelling in a cyclic network network of four hydrogen bonds in the solid state' Nature 397 241-43 (1999).8.

[24] M. J. Frisch, G. W. Trucks, H. B. Schlegel, G. E. Scuseria, M. A. Robb, J. R. Cheeseman, J. A. Montgomery, T. Vreven, K. N. Kudin, J. C. Burant, J. M. Millam, S. S. Iyengar, J. Tomasi, V. Barone, B. Mennucci, M. Cossi, G. Scalmani, N. Rega, G. A. Petersson, H. Nakatsuji, M. Hada, M. Ehara, K. Toyota, R. Fukuda, J. Hasegawa, M. Ishida, T. Nakajima, Y. Honda, O. Kitao, H. Nakai, M. Klene, X. Li, J. E. Knox, H. P. Hratchian, J. B. Cross, C. Adamo, J. Jaramillo, R. Gomperts, R. E. Stratmann, O. Yazyev, A. J. Austin, R. Cammi, C. Pomelli, J. W. Ochterski, P. Y. Ayala, K. Morokuma, G. A. Voth, P. Salvador, J. J. Dannenberg, V. G. Zakrzewski, S. Dapprich, A. D. Daniels, M. C. Strain, O. Farkas, D. K. Malick, A. D. Rabuck, K. Raghavachari, J. B. Foresman, J. V. Ortiz, Q. Cui, A. G. Baboul, S. Clifford, J. Cioslowski, B. B. Stefanov, G. Liu, A. Liashenko, P. Piskorz, I. Komaromi, R. L. Martin, D. J. Fox, T. Keith, M. A. Al-Laham, C. Y. Peng, A. Nanayakkara, M. Challacombe, P. M. W. Gill, B. Johnson, W. Chen, M. W. Wong, C. Gonzalez, and J. A. Pople, Gaussian 03, Revision B.05, Gaussian, Inc., Pittsburgh PA, 2003.

[25] V. Jelić and F. Marsiglio, 'The double-well potential in quantum mechanics: a simple, numerically exact formulation' Europ. J. Phys. 33 1651-66 (2012).

[26] L. Veguilla-Berdecía, 'Tunnelling in a quartic, symmetric, double-well potential', J. Chem. Ed. 70 (1993).

[27] W. Smith, T. R. Forester, DL POLY 2. 0: A general-purpose parallel molecular dynamics simulation package J. Molec. Graphics, 14136 (1996).

[28] A. K. Rappé, C. J. Casewit, K. S. Colwell, W. A. Goddard III, W. M. Skiff, UFF, 'A full periodic table force field for molecular mechanics and molecular dynamics simulations', J. Amer. Chem. Soc. 114 (1972) 10024-10035.

[29] M. W. Mahoney and W. L. Jorgensen, 'A five-star model for liquid water and the reproduction of the density anomaly by rigid, non-polarizable potential functions, Journal of Chemical Physics, 112 8910-22 (2000)

[30] D. Lankhorst, J. Schriever and J. C. Leyte, 'Determination of the rotational correlation time of water by proton NMR relaxation in $\mathrm{H}_{2}{ }^{17} \mathrm{O}$ and some related results' Berichte der Bunsengesellschaft für physikalische Chemie 83 215-21 (1982). 
[31] Lorentz, H. A. 'Über die Anwendung des Satzes vom Virial in der kinetischen Theorie der Gase'. Annalen der Physik. 248 (1): 127-136 (1881).

[32] K. C. Gross, P. G. Seybold, C. M. Hadad, 'Comparison of different atomic charge schemes for predicting $\mathrm{pK}_{\mathrm{a}}$ variations in substituted anilines and phenols', Internat. J. Quantum Chemistry 90445 (2002).

[33] J. Åquist, 'Ion-water interaction potentials derived from free energy perturnation simulations', J. Phys. Chem. 94 80218024 (1990).

[34] S. I. Lee, J. C. Rasaiah, 'Molecular dynamics simulations of ion mobility. 2. Alkali metal and halide ions using the SPC/E model for water at $25^{\circ} \mathrm{C}^{\prime}$, J. Phys. Chem. 100 1420-5 (1996).

[35] D. A. Morton-Blake, 'The entry of ions into a molecular synthetic channel in a membrane', Diffusion Fundamentals 4 195-135 (2015).

[36] G. Zundel and H. Metzger, 'Energiebänder der tunnelnden Überschuß-Protonen in flüssigen Säuren. Eine IRspektroskopische Untersuchung der Natur der Gruppierungen $\mathrm{H}_{5} \mathrm{O}_{2}{ }^{+}$, Zeitschrift für Physikalische Chemie. 58 (5_6) 225245 (1968).

[37] E. Wicke, M. Eigen and Th. Ackermann, 'Über den Zustand des Protons (Hydroniumions) in wäßriger Lösung', Zeitschrift für Physikalische Chemie. 1 (5_6): 340-364 (1954).
[38] E. S. Stoyanov, I. Stoyanova, F. S. Tham and C. A. Reed, 'The nature of the hydrated proton in organic solvents', J. Amer. Chem. Soc. 130 (36) 12128-38 (2008).

[39] J. S. Hub, M. G. Wolf, C. Caleman, P. J. van Maaren, G. Groenhof and D. van der Spoel, 'The thermodynamics of hydronium and hydroxide surface solvation', Chem. Sci. 5 1745-58 (2016).

[40] See for example, Zh. Chen and S. Ghosal, 'The nonlinear electromigration of analytes into confined spaces', Proc. $R$. Soc. A 468, 3139-3152 (2012); Y. Jiang;, Y. Lił, W. Sun, W. Huang, J. Liu, B. Xu, C. Jin ${ }^{a}$, T. Ma, C. Wu and M. Yan, 'Spatially-confined lithiation-delithiation in highly dense nanocomposite anodes towards advanced lithium-ion batteries', Energy Environ. Sci., 2015, 8, 1471-1479; Y. He, 'NanoConfined Room-Temperature Ionic Liquids for Electrochemical Applications' Virginia Polytechnic Institute and State University (2018).

[41] D. A. Morton-Blake and K. Korpela, 'The trans-membrane mobilities of ions in a synthetic molecular channel under the influence of applied electric fields', Soft Matter 6 558-67 (2010).

[42] D. A. Morton-Blake, 'The simulation of the capturing of sodium ions in a monolayer consisting of polythiophene functionalised with crown ether died chains', J. Mol. Liquids 263 316-23 (2018). 